\title{
Research on Path Optimization with PSO for Unmanned Vehicle
}

\author{
http://dx.doi.org/10.3991/ijoe.v11i8.4878 \\ Li Cai, Jianping Jia, Juan Lei \\ Wuhan Donghu University, Wuhan, China
}

\begin{abstract}
The development of autonomous unmanned vehicles is of high interest to many organizations around the world and path planning is the key point of the navigation for the autonomous unmanned vehicle. Intelligent algorithms have been applied in this field and an essential aspect of unmanned vehicles autonomy is the ability for automatic path planning. In this paper, particle swarm optimization algorithm as one of new swarm intelligent optimization methods is introduced into a path planning for autonomous vehicle, which is constructed of a particle representation methods for vehicle routing problem with fast convergence speed. The results show that the particle swarm optimization algorithm can obtain the solution of the vehicle routing problem quickly and effectively. It is a good method for solving the vehicle routing problem.
\end{abstract}

Index Terms-Path Optimization, Particle swarm optimization algorithm, Wheeled vehicle.

\section{INTRODUCTION}

The route planning problem is a key element of the unmanned aerial vehicle autonomous control module. It allows the unmanned aerial vehicle to autonomously compute the best path from a start point to an end point[1][2]. Route planning generates a path between an initial prescribed location and desired destination having an optimal or near-optimal performance under specific constraint conditions. During the past few decades, extensive work has been devoted to route planning. Several approaches have been proposed for unmanned aerial vehicle route planning, such as Voronoi diagrambased method. However, it is difficult to combine the motion constraints of unmanned aerial vehicle with graphic-based algorithms. In order to take the motion constraints of unmanned aerial vehicle into account during the planning process, some new algorithms were proposed. Nevertheless, when the problem becomes more complex, the time spent to find the optimal or nearoptimal path may increase exponentially. It is generally accepted that evolutionary computation as an efficient optimization technique could solve path planning problems[3][4]. To solve this problem, many path planning methods for vehicle routing have been developed[5]. However, each method has its own limitations in some aspects. In recent years, intelligent soft computing techniques such as particle swarm optimization, genetic algorithm method, artificial neural network and interacting multiple model system are proven to be efficient and suitable when they are applied to a variety of systems[6][7].
Particle swarm optimization (PSO) algorithm and genetic algorithm (GA) are new evolutionary algorithms developed in recent years. Geng put forward an improved hybrid particle swarm route planning method with contraction factor, which was used to change algorithm in the balance of performance order to get a better convergence speed and convergence rate[8]. Mohemmed proposed a modified priority-based encoding incorporating a heuristic operator for reducing the possibility of loop-formation in the path construction process. It could find closer sub-optimal paths with high certainty for all the tested networks[9]. Vincent used the genetic algorithm (GA) and the particle swarm optimization algorithm (PSO) to cope with the complexity of the problem and compute feasible and quasi-optimal trajectories in a complex $3 \mathrm{D}$ environment by using a parallel implementation[10]. $\mathrm{Fu}$ presented a hybrid differential evolution (DE) with quantum-behaved particle swarm optimization (QPSO) for the unmanned aerial vehicle route planning, which combined the DE algorithm with the QPSO algorithm in an attempt to further enhance the performance of both algorithms[11]. In order to find the optimal solution, PSO algorithm uses the iterative method from the view of the random solutions and evaluate the quality of the solution through the fitness. Comparison of genetic algorithm, it is more simple than genetic algorithm because it has no "crossover" and "mutation". It can find the global optimum by following the searched optimal values. Thus it can be seen that PSO algorithm is acceptable to solve routing problem, which has flexibility and collaboration features.

In view of improving the limitations, this paper proposes a path planning for autonomous vehicle based on particle swarm optimization algorithm. Obstacle detecting and avoidance method for autonomous vehicle are implemented with some sensors. Then PSO algorithm for path planning is introduced and the simulation results through the comparison of two kinds of optimization algorithm in the MATLAB software show that the method of introducing PSO into autonomous vehicle is convenient. By this means, the path planning is well optimized in real-time way for vehicle routing problem. The chapters are as follows: Section 2 explains system design of autonomous vehicle based on single chip microcomputer. Section 3 introduces the principle and schematic diagram of the PSO algorithm. Then section 4 gives the simulation results of PSO algorithm by comparison of GA algorithm in the MATLAB software. 


\section{AUTONOMOUS VEHICLE MODEL}

Unmanned automatic vehicle we discussed is mainly composed of a mechanical part and a control part. The mechanical part is mainly composed of four wheels: two front wheels of which are responsible for steering control, two rear wheels of which are responsible for driving. The power part is supplied by the battery. In general, wheeled unmanned vehicle is more convenient for operation. The control circuit based on a single chip microcomputer is consist of four parts: motor drive mode into blocks, obstacle avoidance module, tracking module, fire-fighting module.

The unmanned vehicle can turn the drive motor to adjust the steering by detecting obstacles on the way. And it can realize trajectory tracking by the infrared sensor. After the comparison, analysis and processing of the detection signals, the single chip microcomputer can issue corresponding instruction execution to drive motor, and control the entire operation of the unmanned vehicle.

According to the characteristics of infrared detecting, infrared rays have different reflection on different color surfaces. The unmanned vehicle emits continuously infrared light to the ground. If infrared light meets the white line, it will produce diffuse reflection, and the reflected light is received by the receiving tube arranged on the vehicle. While infrared light meets the black line, the light will be absorbed, and the receiving tube will not receive the infrared light. The single chip computer can determine the position of black according to the reflected infrared light with three photoelectric detectors, which are respectively arranged on the left, middle, right of the vehicle in three directions.

\section{THE PRINCIPLE OF PSO ALGORITHM}

The PSO algorithm has a significant difference from other intelligent computing methods that few parameters need to be adjusted. But the key parameters have significant impact on the accuracy and efficiency. In general, the feasible solution of each optimization problem is to search for a particle in space. Each particle has two parameters, one is position in the search space, the other is flying speed of a particle, which can be represented by the following equations:

$$
v_{i d}=v_{i d}+c_{1} * \operatorname{rand}() *\left(p_{i d}-x_{i d}\right)+c_{2} * \operatorname{rand}() *\left(p_{g d}-x_{i d}\right)
$$

(1)

$$
x_{i d}=x_{i d}+v_{i d}
$$

Where vid is the velocity component of the NO. i particle; $c_{1}, c_{2}$ are the acceleration constants; $\operatorname{rand}()$ is random function of the uniform distribution; $p_{i d}$ is optimal solution component of the NO.i particle, which has been searched; $x_{i d}$ is position component of the NO.i particle; pgd is current optimal solution component of the whole community. Equation (1) determines the trajectories of particles in the population. The first part in Equation (1) is the previous speed, which determines the ability of particle in the new search area. The second part is "cognition", which can strengthen an enhanced learning process. The third part is "the agency", which represents sharing information and cooperation. Schematic diagram of the PSO algorithm is shown in Figure 1.
Specific steps of the PSO algorithm are as follows: Firstly, the parameters about the particle population are initialized, including the position and flight speed of the particles, and the variables are initialized such as current searched optimal solution of each particle, current optimal solution of the whole community.

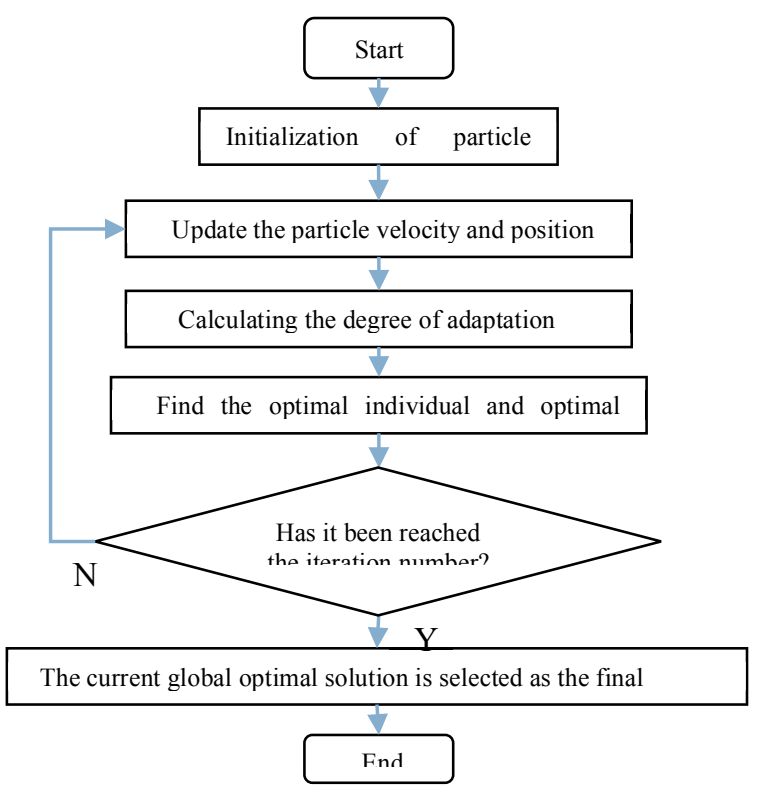

Figure 1. Schematic diagram of the PSO algorithm

Secondly, it evaluates the fitness of each particle. Thirdly, it compares the fitness value of each particles: If the particle is better than current initialized optimal solution of each particle, set the particle to the current value. Meanwhile, if the particle is better than current initialized optimal solution of the whole community, set the particle to the current value. Fourthly, the particle position and velocity are changed according to above the Equations (1) and (2). Lastly, it will automatically end and return to step two if it reaches the end conditions, such as good location or the maximum number of iterations .

In general, the velocity must be limited to the maximum speed. The more maximum speed can improve the global search ability of the algorithm, while the smaller maximum speed can improve local exploitation ability. But if the maximum speed is too high, the particles may fly over the good solution; if the maximum speed is too small, the particle will not be enough to explore in local interval. The maximum speed is usually set to $10-20 \%$ of variable range according to experience, which has a better effect.

Acceleration constants $c_{1}, c_{2}$ represents the statistical acceleration weight of current solution position of each particle and searched optimal solution of the group . So $c_{1}, c_{2}$ is usually set to 2 . Low values will allow particles wander outside the target area before being pulled back. While the high value will cause the particles over the target area suddenly. Population size is generally set to 20-50. which is related to the specific problems. 
PAPER

RESEARCH ON PATH OPTIMIZATION WITH PSO FOR UNMANNED VEHICLE

\section{SimUlation OF PSO ALGORITHM}

Given $\mathrm{n}$ sites and the distances between any two sites, it seeks a shortest path through every site. The graph theory can be described as follows: Given a weighted graph $\mathrm{G}=$ $(\mathrm{V}, \mathrm{E})$, where $\mathrm{V}$ is the vertex set, $\mathrm{E}$ is for the arc set connected with each vertex. The distances between the vertices are known. is the distance between the locations of NO. $\mathrm{i}, \mathrm{j}$. If the variable xij is introduced, the objective function is as follow:

$$
Z_{\text {min }}=\sum_{i=1, j=1}^{n} x_{i j} d_{i j}
$$

Figure 2(a) shows the location layout for path planning simulation, where $n$ is set 24 . And a random path planning is shown in Figure 2(b), following the path $11 \rightarrow 6->3$ $\rightarrow 12 \rightarrow>10 \rightarrow>13 \rightarrow>16 \rightarrow>1 \rightarrow>15 \rightarrow>2 \rightarrow>7 \rightarrow>19-$ $>8 \rightarrow>4->18 \rightarrow>5 \rightarrow>20 \rightarrow>14 \rightarrow>9->17 \rightarrow>11$. The total distance of the random value in the initial population is $184 \mathrm{~m}$. In order to optimize the path, we used two different optimization methods: PSO algorithm and GA algorithm, as shown in Figure 3 and 4 respectively. The total number of places is the same, and the maximum number of iterations is set to 200 respectively in the iterative process, as shown in Figure 3(a) and 3(b).

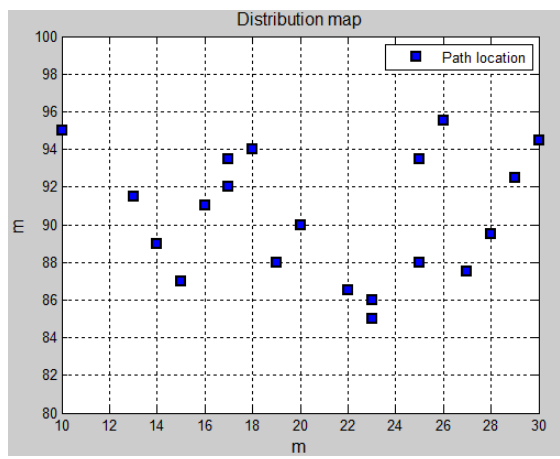

(a)

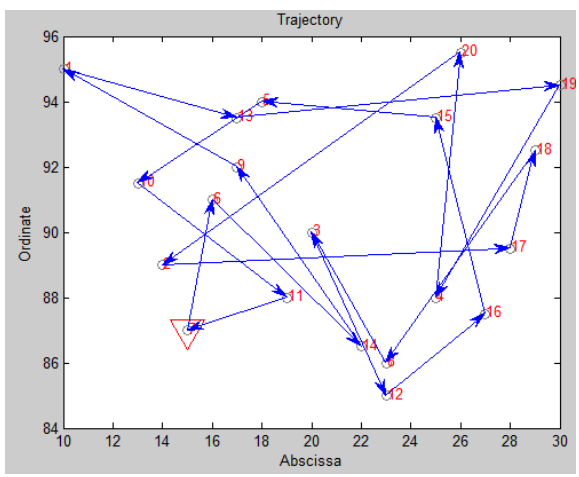

(b)

Figure 2 simulation of path planning in the position layout a- A position layout ; $b-A$ random path planning

There are many possible paths and it need seek the optimal solution by comparing all possible cases. Then the final results about path planning will run out by searching the minimum value according to the Equation (3) by PSO algorithm under the different parameter settings, as shown in Figure 4(a), following the path $10 \rightarrow 1 \rightarrow 6 \rightarrow 9-$ $>13 \rightarrow>5 \rightarrow>15 \rightarrow>20 \rightarrow>19 \rightarrow>18 \rightarrow>17 \rightarrow>16 \rightarrow>4 \rightarrow>8$ $\rightarrow 12 \rightarrow>14->3 \rightarrow>11 \rightarrow 7->2 \rightarrow>10$. Figure $4(\mathrm{~b})$ is the simulation result of path optimal planning by GA algorithm .The optimal distance by GA algorithm is $60 \mathrm{~m}$, following the path $10 \rightarrow>2 \rightarrow>7 \rightarrow>11 \rightarrow>14 \rightarrow>12 \rightarrow>8$ $\rightarrow>4->16->17 \rightarrow>18->19->20->15->3->6-$ $>9 \rightarrow 5->13 \rightarrow>1 \rightarrow>10$. And its total distance is more than that by PSO algorithm.

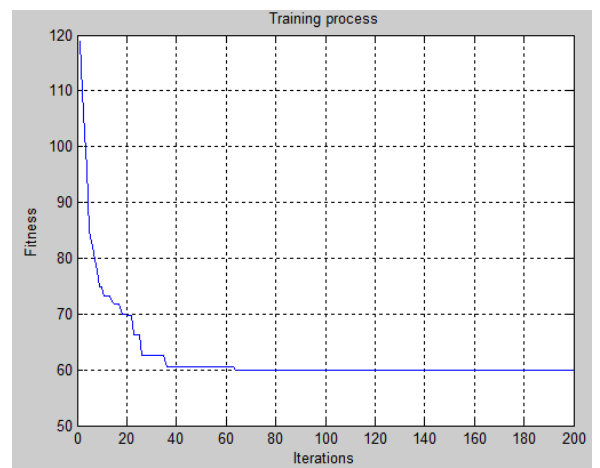

(a)

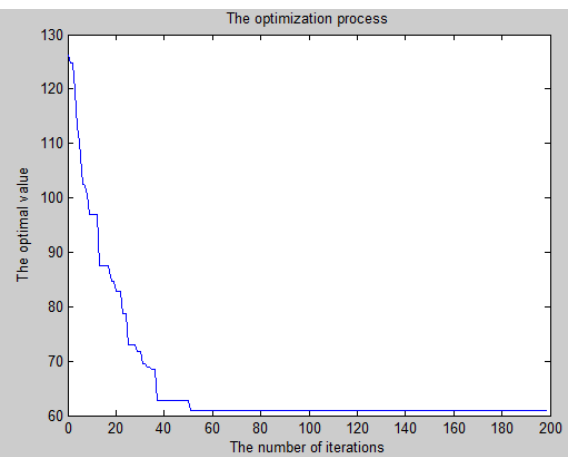

(b)

Figure 3 Iterative process of path planning simulation a- PSO; b- GA b-

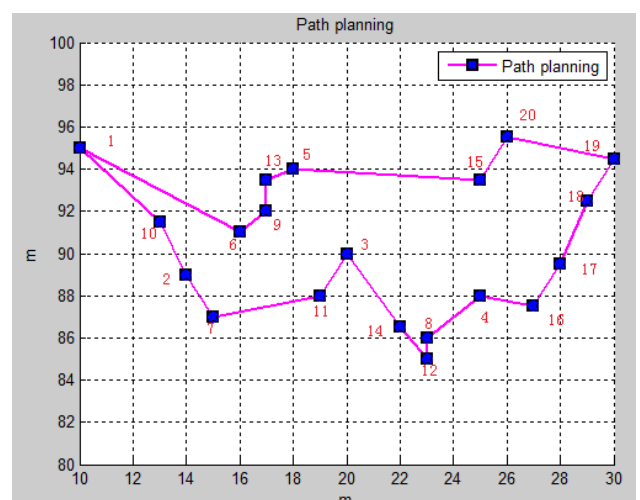

(a)

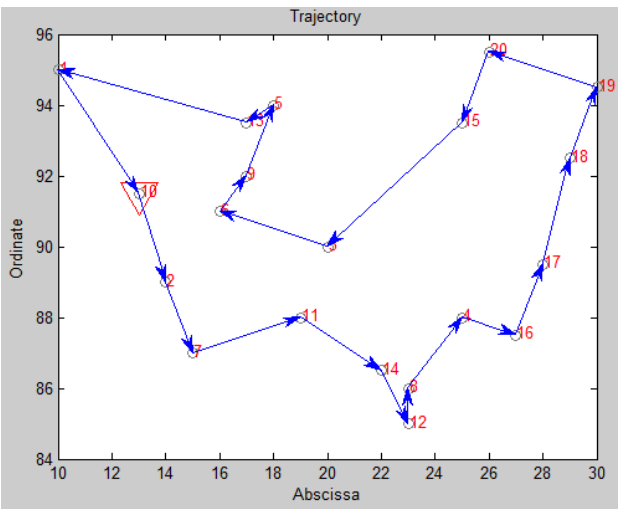

(b)

Figure 4 simulation of path optimal planning a- PSO; b- GA 
Compared with the genetic algorithm, the sharing mechanism of PSO information is very different from that of the genetic algorithm. For genetic algorithm, chromosomes are to share information with each other, so the whole population mobility is relatively uniform to the optimal region. While PSO algorithm is a one-way flow of information, whose search process is to follow the current optimal solution. In most cases, all particles can quickly converge to the optimal solution. No matter from which side, it can draw the conclusion that the system with PSO has better tracking performance than the system without it and the method of introducing PSO into autonomous vehicle is convenient.

\section{CONCLUSION}

This article introduces a simple design of unmanned vehicle based on PSO algorithm, taking a single chip microcomputer as the core, with photoelectric sensors to detect the path goals. Then the principle of the PSO algorithm is introduced, which can find the global optimum by following the searched optimal values. Then the simulation is developed by MATLAB. The simulation results through the comparison of two kinds of optimization algorithms show that the PSO algorithm is more simple than GA algorithm and the introduction of the PSO algorithm into autonomous vehicle has better performance of the path planning compared to the traditional method. Thus it can be seen that PSO algorithm is acceptable to solve routing problem, which has flexibility and collaboration features.

\section{REFERENCES}

[1] Yin, X., Yang, C., Zhou, W.,Xiong, D. 2014. Energy-efficient tracking control for wheeled mobile robots based on bio-inspired neurodynamic. Comput. Inf. Syst. 10(6): pp. 2533-2541.

[2] P. Vadakkepat, O. Miin, X. Peng, and T. Lee. 2004. Fuzzy behavior based control of mobile robots. IEEE Trans. Fuzzy Syst. 12(4) : pp. 559-565. http://dx.doi.org/10.1109/TFUZZ.2004. $\underline{832536}$

[3] Rahim, Nawi. 2008. Path Planning Automated Guided Robot. Engineering and Computer Science, (4): pp. 22 - 24.
[4] Chao,M. 2002. A Tabu Search Method for the Truek and Trailer Routing Problem. Computers \& Operations Researeh, vol.29: pp. 33-51. http://dx.doi.org/10.1016/S0305-0548(00)00056-3

[5] WANG Quan, WANG Wei, etc. 2012. Wheeled robot path planning method based on hybrid strat-egy. Computer Engineering and Applications, vol. 9: pp. 2-5.

[6] Francesco M. Raimondi, Maurizio Melluso. 2005. Robotics and Autonomous Systems. vol.52: 115-131. http://dx.doi.org/10.1016/ j.robot.2005.04.006

[7] Adem Tuncer, Mehmet Yildirim, Kadir Erkan. 2012. A Motion Planning System for Mobile Robots. Advances in Electrical and $\begin{array}{llll}\text { Computer } & \text { Engineering. } & \text { 12(1): pp. }\end{array}$ http://dx.doi.org/10.4316/aece.2012.01010

[8] Geng, Qingbo; Zhao, Zheng. A Kind of Route Planning Method for UAV Based on Improved PSO Algorithm . 25th Chinese Control and Decision Conference (CCDC). 2013: pp. 2328-2331. http://dx.doi.org/10.1109/ccdc.2013.6561326

[9] Mohemmed, Ammar W.; Sahoo, Nirod Chandra; Geok, Tan Kim. Solving shortest path problem using particle swarm optimization. APPLIED SOFT COMPUTING,2008, 8(4): pp. 1643-1653 . http://dx.doi.org/10.1016/j.asoc.2008.01.002

[10] Vincent Roberge, Mohammed Tarbouchi, and Gilles Labonté. Comparison of Parallel Genetic Algorithm and Particle Swarm Optimization for Real-Time UAV Path Planning. IEEE Transactions on Industrial Informatics, 2013, 9(1): pp. 132-141. http://dx.doi.org/10.1109/TII.2012.2198665

[11] Yangguang Fu, Mingyue Ding, Chengping Zhou, and Hanping Hu. Route Planning for Unmanned Aerial Vehicle on the Sea Using Hybrid Differential Evolution and Quantum-Behaved Particle Swarm Optimization. IEEE Transactions on Systems, Man, and Cybernetics. 2013, 43(6): pp. 1451-1464. http://dx.doi.org/10.1109/TSMC.2013.2248146

\section{AUTHORS}

Li Cai is with the Wuhan Donghu University, Wuhan, 430070 China (e-mail: wherec1@163.com).

Jianping Jia is with the Wuhan Donghu University, Wuhan, 430070 China (e-mail: 39557403@qq.com).

Juan Lei is with the Wuhan Donghu University, Wuhan, 430070 China (e-mail: 5091026@qq.com).

This work was supported in part by the Scientific Research Project of Hubei Province(NO.B2013198). Submitted 21 July 2015. Published as resubmitted by the authors 10 October 2015 . 